\title{
45. STELLAR CLASSIFICATION (CLASSIFICATION STELLAIRE)
}

PRESIDENT: O.II. Levalo VICL-PRESIDENT: M.Gerbaldi

ORGA NIZING COMMITTEE: R. Bartaya, D. Egret, C. Garmany, R.O. Gray, M. Grenon, I). Kurtz, J. MacConnell, H. Maehara, P. North

\section{INTRODUCTION}

This report covers research in the field of stellar classification in the period July 1993 to June 1996 . It is divided into several sections which were written by experts in each subfield. I want to thank then for their effort and cooperation. To conserve space, all references are given with only one name followed by $a+$ sign if there are additional anthors.

\subsection{MEETINGS}

During the period covered by this report there was one meeting devoted completely to the sub ject of our Commission: 'The MK Process at 50 Years: A Powerful Tool for Astrophysical Insight". The meeting was sponsored by the Vatican Observatory under the chairmanship of Chris Corbally and was held in Tucson, (ISA) in Sept 1993. The Proceedings of the meeting were published in the ASP (conference Series, vol.60) and the editors are C. Corbally, R.O.Cray and R.F. Ciarrison. Comm 45 has sponsored or so-sponsored the following IAU Symposia or Colloguia during the period:

Symp 162: "Pulsation, rotation, \& Mass loss in Farly-Type stars" Juan-les-Pins Ort. 1993.

Coll 148: " Future Utilization of Schunidt Telescopes", Bandung, Mar 1994.

Symp 166: "Astronomical \& Astrophysical Objectives of Sub-milliarsecond Optical Astrometry", The Hague, Aug 1994.

Symp 167:"New Developments in Array Teclinology \& Applications", The Hague Aug. 1994.

Symp 177: "The Carbon Star Phenomenon, Antalyä, May 1996.

\section{CLASSIFICATION USING SLIT SPECTRA (R.O. Gray)}

\subsection{EAIRI,Y-TYPE STARS}

\subsubsection{Wolf-Rayel and LBV Stars}

Wolf-Rayet (WR) stars both in the Milky Way and in other galaxies (see section2.9) continued to be subjects of intense study during this triennium. Hamannt $(\Lambda \Lambda 113,459)$ presented a visual-region spectral atlas of nearly all single (ialactic: WR stars of the WN sequence. Hamann + (5x.114.003) analyzed these stars using NII'te models, and suggested that the WNli-w subclass continues the WNI. subclass lowards lower luminosities, whereas the WNF-s stars form a separate group with higher temperalures. (rowther+ (633.114.018, 63.114.019, $\mathrm{AA} 304,269, \mathrm{~A} \Lambda 305,541)$ in a series of papers antalyzed a number of WR stars and showed, for instance, that the WNL stars fall into two groups, the WNL tabs and WN7 stars form a continuous seguence in spectral morphology from the Of stars, whereas the WNy stars have some properties in common with IBBV', and they investigated the spectral morphol ogy of Wh stars in the infrared. In addition, they discussed the spectral type of the WN/C star WR8. Nota (ApJS 102,383) found that the Ofpe/WN9 stars form a homogeneous group, between Of stars and B[e] stars. Smith (IAU 163,48) commented on the unsatisfactory state of the classification system for WN stars, and proposed a 3-dimensional system of classification. Kingsburght $(63.114 .026)$ studicd 5 WO WR stars using quantitative spectral-typing criteria. A number of new WR stars in the galaxy have been discovered using various techniques: Davidsont $(58.115 .016)$ classified the spectrum of 11 e $3-519$ as Of/WN9. Coterat (ApJ 461,750) used $\mathrm{K}$-band spectra to show that a cluster, associated with galactic center thermal radio filaments, likely contains up to 13 late WN stars. Blum+ (63.155.095) reported on the discovery of a possible WR at the galactic center, showing similarities to W(9) stars, and Blum-t. (63.114.025) used near-IR spectra of galactic center compact Ile I emission sourres and early-type stars to 


\subsection{N'TI,ASES}

Ayres+ (58.114.081) assmubled an extensive, electronically accessible catalogue of low-dispefsion farult raviolet spectra of chromospheric emission line stars, observed with IUE. Bohlin+ (61.002.048) published a high-dispersion microfiche spectral atlas of $\mathrm{OH}$ stars obtained during the first 10 years of operation of the III L satellite. Talaverat (62.121.136) presented a preliminary catalog of observations of IIerbig $\Lambda \mathrm{e} /$ Be stars obtained with the IUE in the wavelength range from $1200-3300=\mathrm{C5}$.

Walborn+ (63.002.099) published the "International IItraviolet Explorer $\Lambda$ tlas of B-Type Spect.ra from 1200 to $1900=(5, "$ completing the OB natural group to spectral type $B 3$ on the main sequence and 38 at luminosity class Ia, in order to chart the gradual disappearance of the stellar-wind features in normal spectra as a function of spectral type and luminosity class.

Walborn+ (PASP 108, 477) presented an atlas of $27 \mathrm{OB}$ spectra from Copernicus low-resolution (0.2 =C5) data; they demonstrated the pronounced luminosity effect in the S IV II 1063, 1073 wind profiles, and described stellar wind effects in O VI, P V, Si IV, and C III.

\subsection{INFRARED) ('IASSIFIC:ATION}

Volk (5.8.114.134) dikcused the elassification of mid-iufrared spect.ra obtained by IIt AS, with the goal of identifying rare types of spectra. He contrasted the original l,ow Rosolution Spectrometer (J,RS) Atlas classification, the AUTO(:IASS automatic rlassification, and an empirical classification done by cyc. Kwok (58.114.135) proposed a classification scheme for asymptotic branch stars that combines the photospheric spectral type (from ground-based data) with the circumstellar type (from IR $\Lambda$ S data). Walker (62.111.057) used artificial intelligence techniques to derive a new classification system for sources in the IR $\Lambda$ S I.RS $\Lambda$ tlas.

Using IR AS data, Parthasarathy $(58.115 .024)$ concluded that IS II $+34=8326$ is a low-mass, postasymptotic branch B supergiant, and not a massive Population 1 B star located near the outer edgr of the Cialaxy. IIe also (62.115.041) detected a new class of stars from IR AS Point Source Cataloguce (PSC) data. These objects, believed to have cvolved from low-mass stars, liave circumstellar dust shells similar to planetary nobulace, hut their optical spectra resemble those of normal $\Lambda \cdot \mathrm{K}$ supergiants.

\section{Catalogues and Atlases (N.Roman)}

N. Houk reports that Vol. 5 of her reclassification of IID stars will be out early in 1997. She hopes that the remaining volumes can be prepared more quickly.

V. V. Nesterov at al. have prepared a machine-readable version of vol. 112 of the llarvard Annals the portion of the HIDE for which only charts lad been a.vailable. This version includes modern positions and proper motions and references to the General Catalog of Variable Stars and of Suspected Variables when appi priate. (in the $\Lambda$ stronomical Data (enters as 3182).

R. F. Garrison has refined the MK spectral classification system for late 3 stars. He has established a self-consistent sequence of narrow and broadline standirds. Ho has alan recla.saified a number of Bp atara. (A.J, 107, 1550;, 1994)

Other papers and catalogs submitted to the Astronomical Data Centers which may be of interest to spectral classifiers include: $\Lambda$ p JS,09, 135,1995, in which $\Lambda$ bl shows that all highly rotating stars are normal while all very slow rotalors are chemically peculiar. Ap.JS, 86, 453,1993 which classifies stars in a region in ( $V_{n}$ (Macc'onnellt). 3183 Andrillatt atlas of the 837.5-877 $\mathrm{nm}$ spectral region. 3181 'Torres-Dodgen's atlas of infrared spectral of normal stars. 
tra. Mean errors were less than one-half spectral subtype or one luminosity class. They also (61.114.101) discussed the role of Be stars in their ultraviolet spectral classification system. Penny+ (ApJ 460, 906) presented equivalent width measurements of ultraviolet photospheric lines that are useful as spectral type criteria; they showed that ultraviolet spectral typing leads to types that are consistent with optical types.

Slettebak (61.114.102, 62.112.172) used equivalent widths of 16 lines in IUF spectra, plus centroid and edge velocities of Si IV and C IV lines, to study the differences in the strengths and composition of the stellar winds in Be versus the $B$ standard stars.

Niedzielski+ (62.114.119) measured equivalent widths, FWHM and line fluxes for 94 single Wolf-Rayet stars based on low-resolution IUF spectra, which were used for spectral classification in the ultraviolet region and for investigating correlations among the emission lines of different ions. Kingsburgh, Barlow+ (63.114.026) used optical and IUF ultraviolet spectrophotometry of Wolf-Rayet stars to define quantitative spectral typing criteria for WO subtypes from WO1 to WO5.

Hasent (61.114.127) discussed morphological aspects of IIS'T spertra of massive hot stars in the Magellanic (Clouds and M31. McNeil+ (63.114.010) examined the ultraviolet spectral morphology of a sample of B supergiants in the Small Magellanic (loud (SMC). Their analysis included a tabulation of ultraviolet spertral features, evaluation of their variation with spectral type and luminosity class, and comparison with IUF sequences of standard stars.

Vieriat (63.036.330) compared two methods for the automated classification of low-dispersion IUE spectra of normal O3-G5 stars. They concluded that a classification method based on artificial neural networks permits the determination of spectral classes with an accuracy of 1.1 spectral sub classes. Gulati et al. (Bull. Astr. Soc. India 24, 21) devised schemes based on artificial neural networks for classifying IUF low-dispersion ultraviolet spectra. They compared results from a single-level network and from a multi-ievel tree network, and found both to be satisfactory.

\subsection{INIDIVIDUAL, ORJECTS}

'lovmasyant $(58.152 .010)$ continued observations at $1640=05$ with the Glazar space telescope, on board the Mir space station. They identified three groups of early-type stars in the vicinity of Canis Major. They also ( $A J 111,299)$ observed 217 young $O B$ and early $\Lambda$ stars in the direction of the stellar association (ru OB1. From $1640=(55-V$ colors, they derived "latest possible spectral types" for nine of the stars, which had no published types.

Bagnuolot (61.120.016) analyzed the UV photospheric lines of 29 CMa and used a tomography algorithun to produce separate spectra of the two component stars, which they classified as $07.5-8$ lab and $09.7 \mathrm{lb}$, respectively. Penny $+(61.120 .027,61.120 .031)$ reported similar tomographic studies for seven O-type binary stars, while Bagnuolo et al. (61.117.373) used the tomographic technique on i Ori as well as 29 CMa. Thallert $(\Lambda \mathrm{p} J 118,878)$ used this method with high-dispersion IUE spectra to separate the spectra of the Be binary $f$ Per into a B0.5 III-Ve primary and a hot O-type subdwarf secondary.

Hlondel+ (62.112.197) drcomposed the low-resolution IUE spectra of several $\Lambda$ estars from the HerbigBell catalogue into contributions from an F-type emission-line star, a small source of type B9, and a cool disk. Wesemael + $(62.126 .008)$ combined low-dispersion IUE spectra with optical spectra to classify two DA $\mathrm{B}$ stars as unresolved, composite systems.

lleapt (62.114.121) used the COSTAR-corrected GIIRS on UST to study two stars in the dense conter of the 30 Doradus ionizing cluster. They classified R 136a.5 as O3f/WN and R136a2 as WN4-w. Bianchit (62.157.256) classified the supergiant star in N(;(:206-277 in M31 as B1.5 Ia on the basis of a IIV spectrum from FOS on HST, combined with an optical blue spectrum. Drissen+ (AJ 110, 2235) presented spectrograms of 14 individual luminous stars in III) 97950 , the core of the dense galartic starburst NC:C 3603, obtained with the IIST/FOS. They classified three of the spectra as WN6+abs; the others were O3-OS stars. Walborn+ (PASP 107, 104) obtained HST/FOS and ground-based spectra of 18 OB stars in the IMC and SMC, and compared wind chararteristics with spectral types.

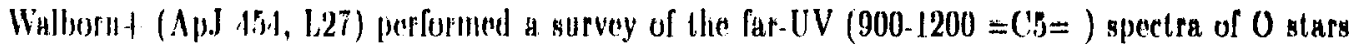
in the Magellanic ('louds, observed with the Hopkins Ultraviolet Telescope (HUT) during the Astro-2 mission. The survey iucluded four 03 stars (one of which is the current candidate for the most massive star known), a pair of OA If supergiants with remarkably different ('NO strengths, and SMC objects with systrmic melal deficiency. 
and luminosity classes $\mathrm{Ia}-\mathrm{V}$, reporting accuracies comparable to human classifiers working with $2 \AA$ resolution photographic spectra.

Gulati+ (ApJ 426, 340; ADASS IV, 25.3) report application of chi-square and $A N N$ methods to 158 digital spectra of $5 \mathrm{~A}$ resolution. Both methods gave similar results, with better than 0.99 correlation between each method and optical classifications.

Vinirat ( $A \& \Lambda S 111$ 383) apply both unweighted motric-dintance mothodn and ANN to nn atlna of 163 low-dispersion IUE spectra of normal stars. They find that both methods give good and strikingly similar results, but the ANN gave somewhat better (mean error 1.1 subtypes) results than the simple unweighted metric distance algorithm (!.4 subtypes).

Von Hippel+ of Cambridge (MNRAS 269, 97) report their application of ANNs to 500 digitized spectra from Michigan Survey plates. Bailer-Jones is continuing this work with an expanded dataset of 5200 spectra and reports (Personal communication) mean ( 1 sigma) errors of 0.86 subtypes when applying a two-layer $\Lambda \mathrm{NN}$ to data "compressed" through principal components analysis (PCA). This represents a considerable improvement over the results of the Cambridge group's earlier one-layer programs. In addition Bailer-Jones reports some progress in luminosity classification, with about 90classifying spectra classified by Houk as luminosity class III or V; he is much less successful in classifying luminosity class IV spectra, to the extent that he suggests that IV may not be spectroscopically distinct from classes III and $\mathrm{V}$ at this resolution. Luminosity classes I and II were too poorly represented in his sample data for

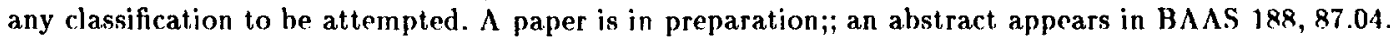

Paisan + ( $\Lambda$ thens meeting on Large Scale Spectroscopy) continue their work investigating the properties of the LMC using low and moderate resolution objective prism spectra. They are supplementing their criterion evaluation methods with $\Lambda \mathrm{NN}$ techniques.

(irosbol (Athens meeting) has analyzed 60,000 early type objective prism spectra in the southern galactic cap using kynthosized colors and hydrogen line photometry.

LaSala \& Kurtz continue application of weighted metric distance techniques to MK resolution slit spectra obtained by Garrison and are exploring a collaboration with Bailer-Jones and the Cambridge group. LaSala presented a review of techniques at Future Possibilities for Astrometry from Space.

'Two masters' theses were produced in the field. (Christlieb (Hamburd) examined low=resolution Schmidt spectra, and Zhu (Toronto) gave a thorough review of the entire field of automated MK classification.

At this point it seems that several methods (ANNs, weighted metric distance, chi-square) are capable of deriving temperature classes with internal and external consistency at least as good as human classifiers working with data of similar resolution. The work of Weaver \& Torres-Dodgen and of Vieira \& Ponz suggests that this is true even for spectra obtained over completely different wavelength regions from the MK standard, a not entirely expected result. No one has yet demonstrated a reliable automatic luminosity classification. The work of Bailer-Jones and of Weaver \& Torres-Dodgen seems currently most promising in this regard. This is clearly the area for further effort.

\section{Classification of Extra-Atmospheric Spectra (J. Rountree)}

The bulk of extra-atmospheric spectral classification continues to be based on data from the International Ultraviolet Explorer (IUE), but the Goddard High Resolution Spectrograph (GHRS) and the Faint Object Spectrograph (FOS) on the Hubble Space telescope (HST) are beginning to be used. Infrared classification is still confined to data from the Infrared Astronomy Satellite (IRAS).

The publication of "The MK Process at 50 Years: $\Lambda$ Powerful Tool for $\Lambda$ st rophysical Insight" (62.012.096) a workshop of the Vatican Observatory held in September 1993, included many papers of interest to specialists in extra-atmospheric spectral classification. Walborn $(62.114 .177)$ reviewed current and nearfut ture ground- and space-based progress for the classification of 03 stars. Corbally (62.114.182) discussed the extension of the MK process to wavelength regions other than from the $\mathrm{Ca} \mathrm{K}$ line to $\mathrm{H} \beta$. Rountreet (62.002.172) reviewed their atlas of B-type spectra from the IU $\mathrm{F}$, based on the ultraviolet classification system. Walborn+ $(62.002 .173)$ reported progress on their atlas of ultraviolet 13-type spectra.

\subsection{METHODOLOGY}

Rount ree $+(B \Lambda \Lambda S 26,1447)$ compared the ultraviolet and optical classificalion of B stars, and concluded that there were no systematic differences, either in temperature type or luminosity class, for 130 - B5. spec- 
hemisphere. See MK50 for more details.

\section{Objective-Prism and Slitless Classification (Jack MacConnell)}

\subsection{IN 'THE GALAXY}

\subsubsection{Geneml and galactir-latitude independent}

During the triennium, two mectings, "The MK Process at 50 Years: a powerful tool for astrophysical

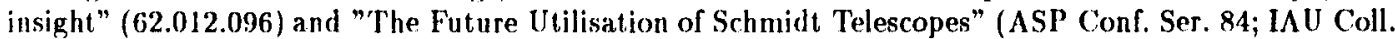
148), wre hold in which a number of review and research papers on stcllar classification and surveys for particular groups of stars were presented.

'The major work in $\mathrm{MK}$ classification from objective prism plates continues to be that of Houk at The Univ. of Mirhigan. She is preparing remarks on her classification of the III) stars that will form Volume 5 of the Michigan Spectral (alalogue which will include stars in the declination range -12 to $+5 \mathrm{deg}$. The hard-ropy version should be distributed in mid. 1997 with an electronic version available some months carlier; betwen $\mathbf{3 3 0 0 0}$ and $\mathbf{3 4 0 0 0}$ stars are included. (lassification has started for Volume 6 which will reach to about $+32 \mathrm{deg}$ and be based on plates taken with both the Burrell (north) and Curtis (south) Schmidt telescopes.

There are many on-going surveys, with both plates and large-format CCDs, behind objective-prisms to search for QSOs and uv-excess galaxies on which interesting groups of stars are found. Examples are the Kiso UV-Excess Survey, the Ilamburg/CfA and Ilamburg/ESO QSO surveys, the KPNO International Spertroscopic Survey for omisaion-line galaxies, the Calan-Tololo Survey, and the Case bow-Diapersion Northeru Sky Survey. There have been many follow-up studies of stars found in this material, e.g. a now PGI159 star with II (Dreizlert, $\Lambda \& A$ 309, 820).

\subsubsection{In the Galactio plane}

Drillingt (PASP 107, 846) have extended the Case-Hamburg OB star survey to $b+/-30$ along the southern galactic plane and found 234 early stars and others with peculiar spectra.

Beerst $(\Lambda \mathrm{J} 108,268)$ presented 158 emission-line objects found in the northern $H \mathrm{~K}$ interference filter/ohjective-prism survey of which 31 have previous identifications; most are late dwarfs or cool, giant variables. Petterson+ $(\Lambda \& \Lambda S 104,233,1994)$ have searched for emission objects towards the HII regions RCW 27, 32, and 33 and the NW parts of the Vela Molecular Ridge finding two new large associations of 'T Tau stars. Kun+ (62.131.171) detected 13 11-alpha emission stars within the area of the dark cloud Lyuds 1340. Bricenot (58.121.011) used the Venezuelan 1-m Schmidt to find a dozen new T Tau stars in the Tan- $\Lambda$ ur star-forming region.

\subsubsection{Out of the plane}

Rodgerst (5x.155.007) presented several hundred early-type stars found on UkS' plates in two orthogonal halo fields. These were classified at $600 \mathrm{\Lambda} / \mathrm{mm}$ at. II-gamma on the basis of the $\mathrm{II}$ and $\mathrm{K}$ and Balmer lines. Pesch + (63.002.062) presented faint, blue stars from plates taken with the Burrell Srhmidt.

\subsection{WORK IN THE MAGHLLANIC: (LOUIDS}

Kontizas + $(62.156 .012)$ have classified luminous stars in $15 \mathrm{LMC}$ associations from UKST plates that give evidence for eontinuous star formation. Kontizas $+(\Lambda \& \Lambda 307,359$ and 308,40$)$ have classified $O B$ stars in a number of I.MC; slellar aggregates, and Morgan (MNR.AS 279, 301) has found a new symbiotic star in the I.MC: of type M4 making it of the latest type and the first M-type donor identified there. Morgant $(\Lambda \& A S 113,539)$ have identified 1185 new ( stars in the outer parts of the SMC from UKST plates.

\section{Automatic Spectral Classification (J. LaSala \& M.J.Kurtz)}

No conferences or workshops forusing on $\Lambda$ utomated Spectral Classification were held between G $\Lambda$ XXII and (iA XXIII. Several important parers have been published in the interval, howver, and the principal groups active in this area report the status of their work.

Weavert $(\Lambda p . J 446,300)$; $B \Lambda \Lambda S \quad 187,19.01)$ extend their application of artificial neural networks (ANNs) to moderate $(15 \Lambda$ ) resolution near-IR spectra to the full range of temperature classes $O$ - $M$ 
(62.117.292) and Barbat (63.117.269) commented on the remarkable long-term changes in the spectrum of the SMC WR system HD 5980. Meyssonnier (63.156.024), in a survey of peculiar emission-line objects in the SMC discovered one possible Be supergiant. Szeifert+ (58.122.057) have found that R40 in the $S M C$ has changed its spectral type from $\mathrm{B} 8$ Ia to $\Lambda 3$ Ia-0 during the past decade. This suggests $R 40$ is the first IBV known in the SMC. Hambly + (61.114.033) obtained spectra of two B-type stars between the Magellanic clouds. Bianchi+ (62.157.256) used both UV and optical spectroscopy of a B supergiant star in M31 to classify this star as B1.5 Ia. Herrero+ (62.157.039) observed a number of the most luminous $\Lambda \& 13$ supergiants in M31 and M33, and have determined their spectral types. Two of the stars are hypergiants. Ocy + (61.132.025) have studied two giant, Ha bubbles in M33, and the single dominant 09 star centrally located in each. Smith $+(\Lambda \Lambda 302,830)$ classified the very late WN star M( $\Lambda 1-B$ in M33 as W N9. McCarthy+ $(\Lambda \mathrm{pJ} 455, \mathrm{~L} 135)$ analyzed spectra of two $\Lambda$-type supergiants in M33; one is clearly metal-poor whereas the other has P ('ygni-like profiles. Mc( Causland + (58.157.266), in a search for young stars in the halo of M31, have identified one object gravitationally bound to M31 that appears to be a G0 star. Giovagnoli+ (61.122.043) presented spectrophotometry of $36 \mathrm{LPV}$ in M33. Most are M supergiants, two are carbon stars; no st rong $S$ stars are seen. Brewer (P $\Lambda$ SP 108,379) discussed a photometric and spectroscopic survey of $A G B$ stars in M31. $\Lambda$ spectrum of the first confirmed S-star in M31 was presented. Drissen+ (62.132.013) deduced from broad stellar emission lines in the optical spectrum of a small cluster in the post-starburst galaxy NGC 1569 the presence of a late-type WN star.

\subsection{GENFRAI}

$\Lambda$ number of spectral atlases were published this trienuium. In addition to those already mentioned, the following are of note: Dallier+ (AASS 116,239) have published a library of H-band stellar spectra, Andrillat+ (AASS 112,475) published an atlas of the infrared spectral region (8375-8770) for O - G0 stars of all luminosity classes and Torres-Dodgen + $(58.114 .025)$ have published an atlas of low-resolution, near-IR. spectra of normal stars, and showed that the stars follow well-defined morphological sequenc's in both temperature and luminosity. Allen $+(63.114 .078)$ presented a grid of stellar classification spectra of moderate resolution in the 5600 - 9000 region, Danks $+(61.114 .037)$ published an atlas of $126 \mathrm{MK}$ southern standard stars in the $5800-10200$ region, and Corral + (MK50) presented low resolution spectroscopy of standard stars in the region 4000 - 9000 . Other papers of general interest include Taylor $(\Lambda \mathrm{pJS} 102,105)$ on the SMR stars, Bo $r(\Lambda \Lambda 305,835)$ on spectral types of objects detected in the ROS $\Lambda \mathrm{T}$ IIRI X-ray error box and Kilkenny ( $\Lambda$ pSS 2.30,53) on various types of hydrogen-deficient stars. $\Lambda$ lso of interest are the following papers: Keenan (MK50) on problems and developments of current spectral classification, with special reforence to Barium, $S$ and Carbon stars, (iray+ (61.114.002) on a 'Trff calibration of $M K$ spectral types through the classification of synthetic spectra, Garrison (62.113.029) and Olsen (Mk50) on the complementarity between photometry and MK spectral classification, Gray (MK50) on the role of precision spectral classification and the definition of localized third dimensions to the existing $\mathrm{MK}$ spectral classification system, Corbally (MK50) on the extension of the MK system to third dimensions and to other wavelength regions, and Meyers-Rice+ (MK50) on a method they have developed to estimate MK spectral types from 10 resolution spectra in the optical region. Walborn continues to work on the spectral classification of $\mathrm{OB}$ stars in this galaxy and beyond; much of his previous work is discussed in sections 2.1.2 and 2.9 above, and most of his ongoing work deals with optical classification of compact multiple systems using HS'T (see below - section 5). He is currently completing a major classification study of 100 stars in the 30 Doradus nebula; further high-quality classifications in 30 Dor will become available from a current program led by $\mathrm{A}$. Moffat. In addition, he is investigating 4 LMC: I supergiants with (NO peculiarities. Corbally and Gray lave just completed spectral classification and analysis of ahout 60 Fiejd $\Lambda$-type HB stars ( $A \mathrm{~J}$ in press), and are embarking on classification and analysis of a number of $\Lambda$-type HB stars in M3, M13 and 92. Abt and Corbally have classified the primaries of 268 possible Trapezium systems. Garrison, as a followup to his 1994 list of Anchor points for the MK System is compiling a list of the most reliable and convenient standards, to be used as Primary Standards of the MK System. Carrison and Corbally are extending their 1993 study of the Halo metallicity gradient with photometric observations and $\mathrm{MK}$ classifications of selected candidate G-dwarfs. Gray has embarked on a study of the physical basis of luminosity classification in the late $\Lambda, F$ and G-type stars. Paunzen \& Gray are currently involved in an extensive search for I Boot is stars botle in the field and in open clusters, and have significantly increased the number of known members of this class. Lloyd Evans has completed observations on his long-running survey of the spectra of about $50 \mathrm{R}$ and $200 \mathrm{~N}$ stars in the southern 
the spectroscopy of suspected variable stars, Castelaz+ (63.122.087) were able to classify Miras $S$ Leo and U Cne at minimum light, Koen+ (MNRAS 277,217) discovered 9 new d Scuti stars, one of which appears spectroscopically to be a 1 Bootis star, and Bernstein + (63.122.092) reported on the discovery of a pulsating field star of spectral type late $A$ or early $F$ which may be a new SX Phe variable.

\subsection{CLUSTERS AND ASSOCIATIONS}

(iarmany (61.152.004) published a useful revinw paper on OB nasociations in which the apectral types of some of the more luminous stars in OB associations are discussed. Spectral types in $O B$ associations and other star-forming regions were published in the following papers: Garcia (58.152.006), 105 stars in the field of Carina OB2; Hodapp+ $(58.131 .299) \mathrm{K}$-band spectral types in L1641 North cluster; Ilughes (62.131.015) Ha emission stars in the Lupus dark cloud complex, and Levato+ (MK50) MK spectral types for 350 stars in the Orion OB1 association, including lists of Am and Ap stars. Many papers include spectral types of stars in open clusters, including the following dedicated to lower main sequence stars: Prosser (61.153.014) in the a Persei cluster; Stauffert (62.153.003) in the Hyades and the Pleiades; Williams+ (62.153.016) late $\mathrm{K}$ and $\mathrm{M}$ dwarf candidate members of Praesepe; Hambly+ (63.153.017) very low mass members of Praesepe, all of which turn out to be $M$ dwarfs; Steele+ (63.153.013) a sample of low mass stars in the Pleiades, and Steele+ (MNRAS 275,841) used low-resolution IR spectra in the study of low mass stars in the Pleiades. Their identification of the two faintest proper motion members as Brown J)warfs is strengthened. Zapatero Osorio + (AA 305,519) studied 7 very low mass stars in a Per, and Bryjat (61.153.001) classified 7 of the lowest mass stars in the IIyades. Other papers dealing with open cluster stars include: Nescit $(\Lambda \Lambda 300,126) 10$ brightest stars in the young cluster NGC 366 ; Beaurhamp+ (62.153.021) B supergiants in the heavily reddened cluster NGC:7419; Harris+ (58.153.020) 80 stars in the field of N(; : 2287; Hillenbrand + (58.153.040) early-type stars in N(iC: 6611; Turnert (61.153.016) spectral types for a number of stars in the cluster associated with the (epheid V1726 Cvgni; Munari+ (MNR $\Lambda S 277,1269$ ) grism spectral types for 5 early-type stars in Bochum 2, a cluster in the galactir anticenter direction. In two interesting papers, Liebert+ (61.153.013) presented optical spectra. of seven faint blue subiwarfs along with one possible post-llB or $\Lambda$ GB manqu star in the open cluster NGC 6791 and letersont (B $\Lambda \Lambda S 27,1438$ ) presented a spectrum of the metal-rich early F-type HB star in NGC 6791. Reimers+ (61.153.036) classified 7 WD candidates in the open cluster NGC 6633; most turn out to be sdo and sdB. Moehler+ (63.154.034) presented spectra of a number of stars along the BIIB of M15; they found that the "blue vertical tail" of the BIIB of M15 consists of stars similar to the HB B-type stars in the field rather than sdB stars. Wenderoth+ $(62.114 .017)$ presented spectra of an extremely blue star in globular cluster NGC 2298. The spectrum appears to be similar to the lle-sdO stars found in the galactic halo. Gray+ $(B \Lambda \Lambda S 26,1489)$ classified a number of A-type HB stars in M3 and M13.

\subsection{STARS IN (BALAXIES}

Walhornt (63.114.039) published an atlas of IIST and CASPEC spectra of 18 stars ranging from 03 to B0.5 Ia, equally divided between the LMC and the SMC. Intercomparisons between stars of the two galaxies were made. Walbornt (58.114.063) have classified two companions of SN $1987 \mathrm{~A}$ in the IMC; both are $\mathrm{B} 2$ although luminosity classifications are not possible. Testor+ $\mathbf{( 5 8 . 1 5 2 . 0 0 8 )}$ presented spectral types for stars in two clustres in OB association LIl 90 in the LMC; the clusters contain WR stars, $O$ stars and an M3 supergiant, and Testor( $1 \Lambda U$ Sym 163,81) observed 6 WR stars in LMC associations LII 101 and LfI 104. ('rowthert (63.114.018) investigated 4 LMC Ofpe/WN9 stars. They revised the lypes of these stars to either WN9 or WN10. Laval+ (62.112.041) presented optical and UV spertral types for the exciting star of N/20 $A$ in the LMC. Heydari-Malayerit (58.153.022) have found that HDF 269828 in the LMC is artually a massive star cluster. They found and classified 8 new $O$ stars. (iummersbach $+(\Lambda \Lambda 302,409)$ presented $U V$ and optical spectra of a number of newly discovered $B[e]$ stars in the LMC: Della Valle ( $(22.066 .016)$ found, from observations of a MACHO lensing-event star that it is a late-F/early G giant in the LMC'. Morgan (MNRAS 279,301) classified N19 in the LMC as an M-type symbiotic star. ('owley+ (62.134.028) showed that the central star of the LMC planetary N66 is beginning to show more pronounced WR chararteristics. Pe at $(61.134 .076)$ presented optical and UV spectra of the central star of PNLM1-64 in the LMC and Pe at (63.134.023) reported on recent spectacular changes in the WR spectrum of the central star of PN LMC-N66. Koenigsbergert 
system is composed of two M3e dwarfs. Cutispotot $(\Lambda \Lambda$ 306,278) discussed spectroscopy of Einstein $X$-ray sources; they turn out to be hinaries with $F$ - $K$ dwarf components. Scarfet $(61.118 .007)$ revised the spectral type of the evolved star in the triple system IIR 6469. Welty+ (63.120.016) monitored the

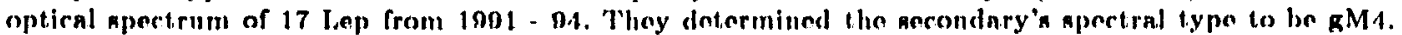
(iizis $+(\Lambda \mathrm{J} 111,365)$ reclassified both components of GJ 1230, Yoon $(61.119 .015)$ derived "quantitative" spectral types for 19 Algol secondaries, Casares+ (58.117.297) classified the secondary of V404 Cyg, a black hole candidate, and Welsh + (MNRAS 275,649) estimaled the spectral type of the mass-donor star in AF Aqr. Dougherty + (MNRAS 280,963) presented a blue spectrum of WR 146, which shows absorption lines attributable to an OB-type companion to the WC6 star. Hambly + (62.118.033) presented spectra of the possible faint companion to PG $0832+676$, a high-latitude B star. The star is early (i, probably a dwarf, and appears to be metal-weak. Griffin + (58.119.001) isolated and classified the individual spectra of $22 \mathrm{Vul}$ and Griffin $+(62.120 .004)$ classified the components of the composite spectrum binary $9 \mathrm{Cyg}$. Simont (61.120.002) wrote on the disentangling of composite spectra of well-detached binaries. (hen+ (MNRAS 275,100) presented spectra of EC11575-1845, a non-eclipsing binary consisting of an sdO primary and late $M$ secondary. Welty $+(A . J 110,776)$ classified the components of V773 Tan as dwarf $K$ PMS stars. Dobrzycka+ $(\Lambda J 111,2090)$ analyzed optical and UV spectra of the recurront nova RS OPH. The cool component is M0-M2, the hot component is probably a B-type star. A number of papers dealt with stars with one or more degenerate components: Vennes+ (62.117.029) Feige 24; Thorstensen+ (62.117.030) EUVE 2013+400; Schmidt (AJ 110,398) GD 245; Jomaron+ (58.120.012) RF1016-05; Napiwotzki + (58.126.014) WI) HZ 43A; Kellet+ (63.116.004) RE 0044+09; licbert+ ( $\Lambda$ pJ 411,424) BE UMa, Hutchings+ (PASP 107,931) new DA WD \& dM binary, and Calal $\mathrm{n}+$ (MNRAS $275,153)$ RE $J 1629+780$.

\subsection{VARIABLF STARS}

Symbiotic stars and cataclysmic variables (CV) dominated the literature dealing with spectral types of variable stars this triennium. Van Winckol+ $(58.002 .051)$ published an atlas of high resolution line profiles of symbiotic stars, and set up a classification system of Ha line profiles in which the starlike (S) and dusty (D) symbiotics are each classified into three spectroscopic subtypes. Medina Tancot (63.117.280) classified a sample of 45 symbjotic stars in the direction of the galactic bulge. Other papers dealing with symbiotic or symbiotic-like stars include: Schmidt $+(61.117 .108)$ barium-rich symbiotics; Van Winckel+ (61.117.073) V417 Cen; Cieslinski+ (62.117.044) and Huang+ (62.117.063) classification in near-IR; Kalert (57.134.051) central star of PN with symbiotic-like spectrum; eiert $(\Lambda \mathrm{J} 110,476)$ MWC: 560 and Guti rrez-Moreno+ (63.117.260) Bl 3-14. Smith+ (Cataclysmic Variables, Kluwer 1994, p 115) discussed a low-dispersion spectroscopic survey of $22 \mathrm{CVs}$, which are classified using spectra into U Gem, Z Cam, SUUMa, pre-cataclysmic, AM Her, intermediate polar (DQ Her), nova-like systems and unknown types. Dhillon+ (MNRAS 275,89 ) presented the results of an IR spectros copic survey of CVs; they used neutral metallic lines from the secondary star to determine their spectral types. Warner ( ApSS 232,89) discussed the spectral types of secondaries of $\mathrm{CVs}$, and how they can be obtained. Other papers dealing with CVs include: Silber (61.117.121) eclipsing nova-like CV, with estimate of spectral type of secondary; (iarnavich + (62.117.249) new magnetic eclipsing (V; Shafter+ (63.117.209) crlipsing magnetic $(V ;$ Drissen $(61.117 .093)$ faint $(V \mathrm{Vs}$; Downes $+(\Lambda \mathrm{J} 110,1824)$ spectra of objects in $(\mathrm{V}$ catalog without published spectra; llakalat $(58.117 .032)$ new $\Lambda \mathrm{M}$ Her system discovered by ROSAT; Buckley+ (58.117.299) new (:V detected by IILAO 1; Osborne (62.117.081) new AM Iler system and Ferrariot (63.117.162) AM IJer system. (layton (PASP 108,225) gave a valuable review of R (;rB stars, including information on their spectral types and the variablitity of their spectra. Ienengagent (61.122.023) discussed the spectrum of the hot R CrB star V348 Sgr, and Benson+ (62.122.001) showed that $\mathrm{Z}$ UMi shows spectral similarities to an $\mathrm{R}$ (rB star. St we+ $(62.121 .118)$ classified the veiled $\mathrm{T}$

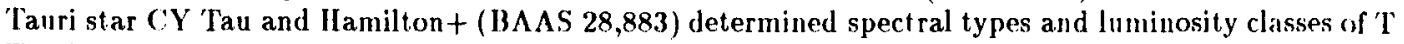
'Tauri stars in the Orion Nebula Cluster. Semi-regular variables were studied by Kerschbanm (58.122.061) who used optical and IRAS low-resolution spectra to separate SRa and SRb stars into groups of defined chemistry - in particular C- and $O$-rich stars, and Koen+ $(63.122 .107)$ classified the red-variable star V 973 Oph. Benetti+ (63.155.130) presented a low-resolution spectrum of a 20 th magnitude star in the direction of the galartic bulge that underwent a microlensing event. This star appears to be a KO IV star. Bragaglia + (63.117.256) studied I,Q Sgr and Wakuda's peculiar variable in Sgr, which they classified as Fis Jab-la with IIa, Itb and the Calcium It triplet in emission. Downest (PASP 10, 134) reported on 
post- $\Lambda$ GB star and Smith + (61.112.069) concluded V18.53 Cyg, a high-latitude B-supergiant, is a hot post-ACB star. Kilkenny + (61.002.209) reviewed the Edinburgh-Cape blue object survey, whjch combines UIIV photometry and low-dispersion slit spectroscopy; objects discovered include hot subdwarfs, WDs, etc. 'Theissen + (57.126.081) classified 18 hot subdwarf stars. Drilling + (61.114.115) discussed follow-up spect roscopy of very hot sdl) stars discovered in the (ase-llamburg-LSU and Beerst objective-prism surveys. Dreizler + (61.114.118) reported on follow-up spectroscopy of stellar candidates in the Hamburg Schmidt survey; in addition to the usual mix of sdO, sdB and WI) stars, the authors discovered two new P(i1159 stars. Ilu+ (57.112.099) classified IRAS 17150-3224, type G2 I, as a PPN, II + (58.133.003) presented optical spectral types of 42 IR $\Lambda S$ selected PPN candidates, Hrivnak (63.112.004) presented the spectra of 6 PPN, all of which show G supergiant spectra. but also features due to molecular carbon and s-process elements. Tamura + (58.134.073) examined the Ha profiles of F supergiants with the aim of identifying PPN candidates and Ilrivnak+ (61.134.012) presented low resolution IR spectra of 16 PPN. Parthasarathy+ (AA 300,L25) found that the central star of the very young PN SAO 244567 was $1 \mathrm{I} I$ in 1971 ; since then it has turned into a PN. Kaler+ (58.134.027) found the nucleus of PN NG(: 2.371 to be an O VI star, on the system of Smith and Aller. Napiwotzki+ (62.134.050) analyzed the spectrum of $\mathrm{BL}+3302642$, the coolest central star known. Tylenda+ (58.134.040) presented classifications for 77 einission-line central stars. Napiwotzki+ ( $\Lambda$ A 301,545) \& Napiwotzki $(58.134 .053)$ classified 38 central stars of old $P N$. They examined more closely the classification of helium and carbon-rich central stars, Other papers dealing with PNN include: Acker+ $(\Lambda A 305,944)$, central star of M1-25 = [WC. 6]: Harringtont (58.134.013) PNN of IR AS 18333-2357 in M22 resembles an sdO star; Leuenhagent $(61.114 .026)$ V348 Sgr = [WC 12]; 'Tweedy + (62.134.016) the displaced central star of Sh 2-17.1; Hebert (58.134.052) K648, PNN in M15; Kondrat'eva (62.134.009) classified 8 central stars of low-excitation PN and Kondrat'eva (58.134.074) studied the rapid evolutionary changes of Th 4-4. Kelly+ (63.114.077) presented near IR spect ra of a sample of evolved stars ranging from Mira variables to PN; an evolutionary sedyumere is visible. Finally, the papers doaling with the discovery and classification of WDa are nimont too numerous to mention. Of note is Liebert (MK50) who gave an historical review of the develnpment of spectroscopic classification schemes for WDs, and suggested changes to the presently used system. Wesemael+ $(58.126 .009)$ published an atlas of optical spectra of WD stars, including all major spectral rlasses and subrlasses and discussed the spectral classification system for WDs. Barstow+ in a series of papers (61.126.058, 61.126.059, 62.126.066, 63.117.151,63.117.192, MNRAS 277,971) reported on the discovery and classification of a mumber of WDs, many as a result of ROS $\Lambda$ ' observations. Included was the hottest known highly magnetic D $\Lambda$. Wernert $(63.126 .043)$ reported on the discovery of two hot DO white dwarfs exhibiting ultra-high-excitation a Absorption lines, possibly prototypes of a new DO subclass. Other papers on the discovery and/or classification of WDs include: Darling $+(\Lambda \mathrm{J} 111,865)$, Darlingt $(62.114 .158) \&$ Wegner+ (58.114.004) presented spectral types for stars from the Kiso UV excess survey - the stars include subdwarfs and WI)s; Everett+ (PASP 107,1059) 124 objects from Case Lowdispersion Northern Sky Survey; Mason + (63.142.075) spectroscopy of EUV sources from the ROSAT Wide Field (amrra; Jordan + (57.126.068) type I $\Lambda$ 13; Danzinger+ (58.117.038) type I)C as a companion of a pulsar; Wernert $(\Lambda \Lambda 284,907)$, hottest known He-rich WD; Reimers+ (61.126.042) new magnetic 1)A; Dreizlor+ (61.126.067) hot $\mathrm{J})($ ) Dreizler+ $(\Lambda \mathrm{A} 303, \mathrm{L53}) 4$ hot WDs with ultra-high excitation features in their spectra; licbert $+(61.126 .025) \mathrm{C}$ star with WD companion and a highly magnetic degenerate star; Koestert (61.126.041) new spectra of DAB prototype GD 323; Liebert+ (61.134.037) DA nucleus of I'N WIIIS 1; Wescmael+ (62.126.008) two hybrid DAB stars; van Kerkwijk+ (ApJ 45.,L141) WI) companions of radio pulsars; Craig + (AJ 110,1304) \& Craig + (BAAS 28,945) spectral types of WDs from EUVE surveys; Ruiz (A.J 111,1267) 6 new cool degenerate stars; Tytler+ $\mathbf{5 8 . 1 5 9 . 0 0 8 )}$ IV excess objects and Lamontagne+ (61.126.192), Feige 55. Cowley+ (PASP 107,927) reported on the discovery of a new hot PG 1159 star and Hambly $+(\Lambda \mathrm{pJ} 448,628)$ identified a degenerate star in the halo of our galaxy.

\subsection{BINARIES AND MULTIPLES}

Binary stars, ranging from chromospherically active stars to degenerate pairs were the subject of artive research this trienniem. Fekel+ concentrated mostly on chromospherically active stars in binary systems: (57.116.019) HID $212280 ;(58.120 .008) \mathrm{g}$ ( $\mathrm{fem} ;(58.120 .016)$ chromospherically active giants; $(62.120 .002)$ AIS $11060 ;(62.116 .005)$ HI) 30957. Bopp + (58.116.063) studied an extremely active RS CVn star; Jeffriest $(58.116 .078)$ studied a chromospherically active late-type binary system, detected by ROSAT; the 
region. Kirkpatrick+ (61.118.022) pres ented spectral types of components in low-mass binary systems in order to find a mass-spectral class relationship for cool dwarfs. Jones+ $(61.114 .010)$ presented an IR. spectral sequence from $\mathrm{M} 2 \mathrm{~V}$ to beyond $\mathrm{M} 9 \mathrm{~V}$, showing the importance of $\mathrm{H} 2 \mathrm{O}$ bands, which are used in a temperature calibration, Baraffe+ (ApJ 461,L51) derived a mass-spectral class relationship for M dwarfs, and Leggett $+\left(\Lambda_{p} J S 104,117\right)$ published an atlas of low-resolution IR spectra for red dwarfs which they used to establish a temperature scale. The following papers presented new spectral types for cool dwarfs: Soderblom + (ApJ 460,984) and Fekel+ (58.114.108) HD98800; icela+ (63.117.270) dMe; Mathioudakis + ( $\Lambda$ A 302,422) dMe; Jeffries+ (AA 305,L45) active K2 V; Martin + (AASS 116,467) 63 late dwarfs; Fekel (63.116.065) HD181943; Broshh+ (61.111.011) uncataloged dM3; Ruiz+ (63.114.085) high proper motion $\mathrm{dM}$ and cool degenerates; Jeffries+ (62.116.016) rapidly rotating $\mathrm{dK}$ and Jeffries+ (62.116.071) dMe. Brown dwarf (BI) candidates also received much attention: Mart $n+(62.115 .041)$ presented spectra of $10 \mathrm{BD}$ candidates in the general field; st rong $\mathrm{Li}$ absorption was found in all 10 . Basrit $(\Lambda \mathrm{pJ} 458,600)$ published high resolution spectra of PPL 15, a BD candidate; the authors discussed its spectral type and noted that it shows the lithium absorption feature. Iannat (63.115.008) reported on the parallax and spectral type of BD) candidate ESO 207-61 and Mould+ (61.114.048) presented a spectrum of the B1) candidate P(C025+0447, more likely a late $\mathrm{dM}$. Rebolot $(63.114 .098)$ discussed the ability of the presence of $\mathrm{L} i$ absorption to confirm BI) status and summarized the search for $L i$ in many $B 1$ ) candidate s. Finally, this triennium saw the confirmed discovery of two brown dwarfs. Rebolot (Nature 377,129 ) announced that Teide 1, a low-mass star in the Pleiades was clearly substellar. An optical spectrum was presented and given a spectral type of M9, although the lithiun feature could not be detected in the 6 resolution spectrum. Oppenheimert (Scincuce 270,1418) presented an Ih spectrum of the cool brown dwarf (iliese 22913 (identified as a BI) by Nakajimat, Nalure 378,463) which had strong afliuities with the spectrum of Jupiter, in particular methane absorption features, leading to a Tsurface $1000 \mathrm{~K}$. Other papers concerning the classification of late-type stars include: Bhatt $+(62.114 .029)$ SAO75669, gM; Groenewegen + $(\Lambda \Lambda 305,475)$ AFGL 2477 - likely a composile spectrum M6S+C; Kipper+ $(\Lambda \Lambda 306,489)$

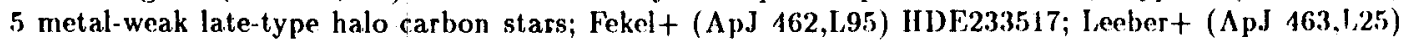
IRAS 13568-6232 = M6-8 III; Stpie+ (58.116.097) IIR1362; Chan (58.112.069) J stars; (ohen + (AJ

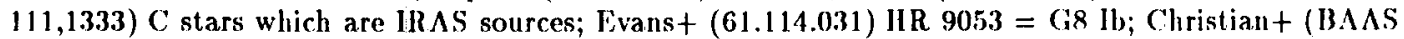
$28,941)$ late-type stars from Hosat detections and Winfrey (BAAS 26,1382) cold IR $\Lambda$ S supergiants.

\subsection{PRE-MAIN SEQUENCE STMRS}

Meyer (BAAS 26,1471) described a program of spectral classification in the J,11\& $\mathrm{K}$ bands of YSOs, beginning with observations of many $M K$ standards in these bands. Pravdo $(\Lambda p . J 417,342)$ classified 10 new $K$ and M PMS stars which are X-ray sourres in the Orion star formation region, Greenet $(\Lambda p J$ 450,233) presented spectral types of YSOs in the $r$ Oph dark cloud (1.1688), Kenyon + (62.121.003) rlassified optical spectra of $60 \mathrm{~mm}$ IRAS sources in the 'Tau-Aur molerular rloud; 9 are probably PMS stars. Simon $+(58.121 .065)$ classified $T$ 'Tauri stars in PMS binary systems and Strom + (58.121.039) reported on the discovery of two FU Orionis objects in L1641. Walter+ (61.152.001) reported on followup spect roscopy of 28 low-mass PMS stars as counterparts of Einstein X-ray sources in the Upper Sco OB association. Jura $+(63.112 .137)$ concluded from optical spectra that JlR479613 is a PMS M2 star. 'Th + (61.002.025) published a new catalog of Herbig Ae/Be stars. Hillenbrand+ (63.131.241) determined the spectral types of stars associated with two Herbig Ae/Be stars. The following papers report the discovery and classification of new Herbig Ae/Be stars: Zavagno+ (61.121.006) YSO in S187; Aspint (61.121.011) V1318 Cyg; Hessmant (AA 299,461) LkHa 349; Whitney (58.121.061) \& Claytont (57.121.088) Z ('Ma;

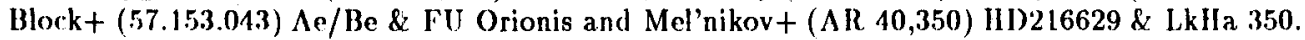

\subsection{HIGIIIY EVOLVED STARS}

Compared to the previous triennium, the research into highly evolved stars - meaning for the purposes of this survey, post-AGB stars, sdO and sdB stars, proto-planetary nebulae (PPN), planetary nehula muclei (PNN), and white dwarfs (WI)) - horizontal branch stars are covered in other sections - has increased dramatically. Parthasarathy (MK50) reviewed the characteristics of post- $\Lambda$ (iH supergiants, Kendall (62.114.075) classified IID 177566 as a low-mass, lot post-AGB star, Rierat $(\Lambda \AA .302,137)$ found the spertrum of IRAS 17123-1755 dominated by emission lines with a few alsorption lines, and concluted it is a massive post- $\Lambda$ GB star, Van Winckelt $(\Lambda \Lambda 306, \mathrm{~L}, 37)$ found III) 187885 , to be $\Lambda 2 \mathrm{lb}$, and a likely. 


\subsection{A-ANI) F-TYPE, STARS}

Abt+ (ApJS 99,135) published new MK classifications for $2000 \mathrm{~A}$-stars in the BSC. The authors used the data to examine the relationship between rotational velocities and spectral peculiarities in A-type stars. They also found that many "normal" stars near $\Lambda 2$ IV have spectral peculiarities. Rao (MK50) discussed these A2 IV stars. Torres-Dodgen (MK50) presented a 15.5 resolution near-IR spectral atlas of A-type stars, while Abou Elazm (58.114.077), Carquillat+ (62.120.003) and Carquillat+ (63.120.014) studied $\Lambda$-type composite spectra. Considerable offort has been expended on the $\lambda$ Bootis stars this triennium. Gray $+(58.152 .003)$ discovered one $\lambda$ Bootis star in Ori OB1 plus 9 metal-weak A-stars in Orion OB1 and Lacerta OB1. Levato+ (MK50) as well found $3 \lambda$ Bootis in Orion OB1. Strenburg (58.114.027), in the process of analyzing most of (iray's list of $\lambda$ Bootis) discovered a $\lambda$ Bootis in a binary system and was able to confirm the classification of $\lambda$ Bootis stars into peculiar and normal hydrogen-line classes. Ulevt (58.114.079) also examined the hydrogen line profiles of the $\lambda$ Bootis stars. Holweger $+(62.114 .030)$ inestigated the 1600 absortion feature often used as an UV criteron for membership in the $\lambda$ Bootis class and found it is due to a quasy molecular satellite of Lyman $\alpha$; this feature is therefore not seen in normal $\Lambda$ type spectra only becasue of the normally heady line blanketing . Andrillat+ $(\mathrm{AA} 299,493)$ examined the behavior of the 1 Bootis stars in the IR. North $+(62.122 .014)$ showed that HD37151 is not an Ap star and Catalano (MK50) discussed the spectral classification of upper MS chemically-peculiar stars. In the course of investigating $F$ strong $\$ 1077$ stars, North $+(61.114 .007)$ discovered 8 barium dwarfs. Jones+ (58.115.015) classified IRC +10420 as a very luminous $F$ supergiant (F8 Iat) with emission lines. Gulliver+ (62.114.009) using high dispersion, high S/N spectra confirmed the suggestion that Vega is a rapid rotator seen pole-on. Coulson + (63.112.136) classified a star in a dusty, organically rich environment to be F8 V. Kurtz+ (MNRAS 276,199) classified HD 40765 as a 5 Puppisstar, and found that it is a d Scuti variable. Denizinan+ (58.112.098) described the spectrum of a stable shell star. De Medeiros+ (61.120.022) discussed the spectral type of HD101841. Hambly + (AA 306,119) classified stars around a Btype halo star, and Flynn + (63.002.108) presented a catalog of faint blue stars at medium to high galactic latitudes, and classified them as BHB or non-BHB stars. Bell+ $(61.115 .205)$ have obtained spectral types for $34 \mathrm{B9}$ to A5 stars at the NGP. Wilhelm+ (61.114.121) described a method to distinguish Field HB (FIIB) stars from normal A-type stars. Corbally+ (61.114.120) discussed the appearance of FHB spertra. Lu (61.155.203) reported on a survey for F and G stars at the South Galactic pole and Garrison (61.114.130) discussed the $\mathrm{MK}$ classification of yellow supergiants in the halo.

\subsection{LA'TE-TYPE S'TARS}

Kenan (58.111.031) has published a revised MK classification scheme for red carbon stars, combining some of the features of the old $\mathrm{R}, \mathrm{N}$ and $\mathrm{C}$ classifications with numerical abundance indices and Keenant (62.115.018) reviewed spectral classification of SMR, strong-CN and R stars. Barnbaum (61.002.007) has published a ligh-resolutiion atlas of Carbon stars. (inestet+ $(62.114 .115)$ studied cool MK standards in the $8.380-8780$ region, Origliat $(58.158 .102)$ presented indices for spectral classification of cool stars in the 1.5-1.7 mm region, Mallik (61.11..015) found the Ca triplet lines to be strongly correlated with gravity and metallicity in cool stars, $\Lambda$ lit $(\Lambda \mathrm{J} 110,2415)$ presented an atlas of $2 \mathrm{~mm}$ spectra of cool dwarf stars, and MacConnell+ (MK50) discussed the classification features of cool luminous stars in low resolution spectra. Hammersley + (61.114.008) demonstrated that near-IR CVF spectroscopy can be used to determine accurately the spectral type of $K$ and $M$-type stars. Garrison + (58.114.075) classified 299 faint early-G dwarf candidates in the direction of the galactic poles. Reid+ $(\Lambda \mathrm{J} 110,1838)$ presented spectral types of 1746 stars in the 3rd catalog of Nearby Stars. Carballo+ (MNRAS 277,1312) derived spectral types for the brightest sources optically identified as ROSAT X-ray sources. These objects range from F7 to M1. 'The study of lower MS stars made important advances this triennium. Kirkpatrick+ (63.114.068) presented spectra for 20 dwarfs later than $M 7$, and used them to set up a spectral classification scheme for types M7 to M9, Kirkpatrick (63.114.097) published a spectral sequence from K5 to M9 and Simonst (58.114.090) reported on preliminary results from an IR survey with the purpose of establishing a spectral sequence from MO - M10+ in the IR. 3 dwarfs were found to be later than M9 and have enhanced VO bands. Ilenry+ (62.114.011) presented spectral types for all known northern late dwarfs closer than 8 pc, Kirkpatrick + $(58.126 .117)$ reported on a program to recognize missed low-luminosity dwarfs in the solar vicinity and Kirkpatrick + (62.115.026) assigned spectral types to color-selected targets from a dcep (CD) survey for cool dwarfs. Boeshaar+ (MK50) discussed the spectroscopic properties of $M$ dwarfs in the $2 \mathrm{~mm}$ 
show that these stars are OB giant/supergiants, WC or WN7-8 stars. Figer $+(\Lambda p .147,1,29)$ discovered two new WR stars and an JBV in the quintuplet. AFCiL 2004 near the galartic center. Wjlliamst (MNRAS 275,889) presented uear-IR spectra of two IRAS soures; one turns out to be a W'(8-9) star, whereas the other is a luminous emission-line object, similar to h Car. New spertra of individual stars have yielded new rlassifications: Smith $+(61.122 .013)$ reclassified He $3-519$ and $\Lambda$ G Car as WN 11 instead of Ofpe/WN9, Nota (57.156.101) speculated, on the basis of new echelle spectra that S119, classified previously as Ofpe/WN9 is a new LBV, and Niemelat (63.120.027) have reclassified HIDF320102 as an $\mathrm{O} 6+\mathrm{WN}$ binary system. Ilumphreys $+(62.122 .031)$ presented a review paper on LBVs, including a useful description of their spectral characteristics.

\subsection{2. $O$ - and B-type Stars}

Walborn (MK50) discussed the spectroscopic criteria which distinguish the 03 class in both the optical and $U V$ wavelengths and has presented arguments which may lead to the definition of an $\mathrm{O} 2$ spectral class. Ilanson + (61.114.046) presented an atlas of $2 \mathrm{~mm}$ spectra of $O$ and early B MS stars, which they used to set up a classification system useful in the investigation of hot stars embedded in shrouded II II regions. They applied their new classification system to $30+$ stars in the region of M17 (BA SS $26,1390)$ and identified 7 new $O$-type stars. Ianson $+(\Lambda$.JS in press) present an expanded spectral atlas of $180 \mathrm{OB}$ stars with a further discussion of their classification system. Conti+ (63.114.043) presented K-band spectra of two Of stars which show emission-line morphology similar to WN stars. Vijapurkart (58.114.084) classified 291 stars identified as OB+ in the Case- Hamburg Luminous Star survey. Parker (58.152.001) presented new classifications for 54 stars in the 30 Doradus OB association; 24 of these stars are O-stars, and Walborn (BAAS 28,931) discussed the results of a number of spectral classification programs in 30 Doradus. Drilling (M K50) discussed his work in the discover y of more than 400 new $O$ and I supergiants plus a number of astrophysically important peculiar stars through spectral classification. Reed+ (63.002.034) published a spectroscopic database for Stephenson-Sanduleak southern luminous stars. A total of 6182 classifications are listed. Undorhill (57.120.027) discussed the spectral types of the components of Plaskett's star, Underhill (61.114.011) argued that the spectral type of IID 108 is O7fpe III, and is related to LBV and B[e] stars and Gies+ (58.118.018) showed that the secondary spectrum of 15. Mon gives a spectral type of $09.5 \mathrm{~V}$ for the companion. Bakker+ $(\mathrm{A} \Lambda \mathrm{307,869)}$ studied the peculiar supergiant IID)101584, and concluded that it is not an F supergiant, but a B star with a strong stellar wind. Kapert $(\Lambda \Lambda 300,416)$ revised the spectral type of the $B 1$ la + companion of an $X$-ray pulsar, Gies (61.120.012) gave a spectral type for the B-type companion of HD 53975, and Coe (62.142.018) reported that the optical counterpart of GRO J1008-57 is an ORe star. Littlet $\left(\Lambda_{\mathrm{pJ}} 447,783\right)$ derived basic parameters and spectral types for 6 blue stars in the galactic halo, Moehler+ (61.114.013) investigated PG 2120+062 and found it to be an apparently normal O9 star at high galactic latitude, Hebert ( $\mathrm{AA}$ 300, L33) have classified HS $1914+7139$, an apparently normal B star far above the galactic plane as B2.5 IVn, Schmidt + (AA 306,L33) discussed the spectrum of PG 0009+036, another rapidly rotating normal B-star in the galactic halo, Hambly+ (58.114.104) classified 6 high galactic latitude, presumably young, stars, and Kilkenny+ (MNRAS 276,906) presented classifications for 30 apparently normal high galactic latitude B stars; 11 appear to be normal B stars, the others are likely subdwarfs. Mitchell+ $(61.114 .114)$ gave preliminary classifications for a new sample of faint halo B stars. Dufton+ (58.119.021) classified the components of the high-latitude B-type binary SU Psc. Garrison+ $(61.114 .022)$ refined the MK system for the late B-type stars and presented spertral types for 200 stars on the revised system. Garrisont (BAAS 27,1311) evaluated the (: II 4267 line as a suitable spectral classification criterion.

\subsubsection{Be and Shrll Stars}

(stet (58,112.003) reported on the discovery of 5 new Be stars and oue new Oe atar. Autrillat (61.112.022) studied 74 Be stars in the near IR, and noted a correlation of P7 emission with spectral type. The optical counterpart of a softg-ray repeater was revealed by van Kerkwijk+ (63.143.167) to have a spectrum similar to that of a B[e] star. Lawson+ (61.117.020) showed that IID 183133 is a Be interarting binary. Menchenkova (5, 112.101$)$ reported on the spectrum of Pleione during 1990, and Singht (61.112.22:3) reported on the spectrum of $f$ Per over an interval of 18 years. Denizmant (62.112.286) described the spectrum of the Be/ Shell star V923 $\mathrm{Aql}$. 